\title{
AVALIAÇÃO NUTRICIONAL E CONSUMO ALIMENTAR DE ATLETAS DE RUGBY
}

\author{
Liégi Tajana FERRANTI ${ }^{1}$ \\ Cíntia Reis BALLARD ${ }^{2}$ \\ Indiomara BARATTO 3 \\ Daiana NOVELLO ${ }^{4}$
}

\begin{abstract}
${ }^{1}$ Nutricionista, Universidade Estadual do Centro-Oeste (UNICENTRO). liegi_fe@outlook.com
${ }^{2}$ Nutricionista, Mestra, Doutoranda em Alimentos e Nutrição (UNICAMP). cintiareis@ hotmail.com

${ }^{3}$ Nutricionista, Mestra, Doutoranda em Obstetrícia (UNIFESP). indy@ hotmail.com

${ }^{4}$ Professora, Doutora, Departamento de Nutrição (UNICENTRO). nutridai@gmail.com
\end{abstract}

\section{Recebido em: 14/01/2014 - Aprovado em: 03/06/2015 - Disponibilizado em: 15/07/2015}

RESUMO: O objetivo do estudo foi avaliar o estado nutricional e o consumo alimentar em atletas da equipe de rugby de Guarapuava, PR. Vinte e um atletas com idades entre 19 e 37 anos foram submetidos à avaliação antropométrica (peso, altura, índice de massa corporal - IMC, circunferência do braço, pregas cutâneas e bioimpedância) e à anamnese nutricional (frequência alimentar e dia alimentar habitual). A porcentagem média de gordura corporal foi acima de $17 \%$. A média de taxa metabólica basal foi de $1.921,77 \pm 171,23 \mathrm{kcal}$ e do gasto energético total de $3.418,10 \pm 321,63 \mathrm{kcal}$. Carboidratos, cálcio, vitaminas A e $\mathrm{C}$ obtiveram valores abaixo da recomendação $(\mathrm{p}<0,05)$, assim como as calorias. Valores de proteínas e ferro se mostraram superiores pelo elevado consumo de carnes, leguminosas. Conclui-se com base nas inadequações de ingestão de nutrientes e de composição corporal que intervenções nutricionais são necessárias, visando a melhora do desempenho esportivo e a redução de riscos à saúde nesses atletas.

Palavras-chave: Atletas. Composição Corporal. Esporte. Hábitos Alimentares. Micronutrientes.

\section{Nutritional assessment and food consumption of rugby athletes}

ABSTRACT: The objective of the study was to evaluate the nutritional status and food intake in rugby athletes team Guarapuava, PR. Twenty-one athletes aged between 19 and 37 years underwent anthropometry (weight, height, body mass index - BMI, arm circumference, skin folds and bioimpedance) and dietary assessment (food frequency and usual daily diet). The mean percentage of body fat was above 17\%. The average basal metabolic rate (BMR) was $1.921 .77 \pm 171.23 \mathrm{kcal}$ and total energy expenditure (TEE) of 3.418.10 $\pm 321.63 \mathrm{kcal}$. Carbohydrates, calcium, vitamins A and $\mathrm{C}$ values were below the recommended level $(\mathrm{p}<0.05)$, as well as the calories. Values of protein and iron were higher than the high consumption of meats, legumes. The conclusion is based on nutrient intake and body composition inadequacies that nutritional interventions are needed in order to improve the sports performance and the reduction of health risks in these athletes.

Keywords: Athletes. Body Composition. Sport. Dietary Habits. Micronutrients.

\section{INTRODUÇÃO}

O rugby clássico é um esporte de intenso contato corporal e apresenta um aumento expressivo no número de praticantes nos últimos anos (CONFEDERAÇÃo
BRASILEIRA DE RUGBY, 2014). Um time de rugby é composto por 15 jogadores, divididos basicamente em dois grupos que desempenham funções distintas: os Forwards, considerados defensores da equipe, com atributo principal de força; e os Backs atuando 
como marcadores de pontos, tendo como principal requisito a velocidade. O período de uma partida oficial é de dois tempos de 40 minutos com intervalo de 10 minutos (LOPES et al., 2011).

Essa modalidade esportiva exige inúmeras respostas fisiológicas dos jogadores, por se tratar de corridas de alta intensidade e também da frequência de contatos. Em uma partida existirão necessidades específicas para cada indivíduo, de acordo com o condicionamento físico e os níveis de treino (PERRELA et al., 2005). As respostas ao exercício estão diretamente associadas a variáveis como intensidade, duração do esforço e treinamento, além do estado nutricional do atleta (PANZA et al., 2007).

Os desgastes nutricionais que são gerados pelo esforço físico podem acarretar problemas à saúde se o déficit energético e protéico não forem supridos adequadamente. Assim, a alimentação torna-se essencial para o bom desempenho dos participantes e, imprescindível para suportar os períodos de treinos e competições.

A capacidade de rendimento possui forte relação com a ingestão equilibrada de nutrientes. Somente através de um adequado planejamento alimentar, se consegue atingir as necessidades individuais dos atletas (CABRAL et al., 2006; OLIVEIRA; MARINS, 2008). A avaliação antropométrica é relevante para o sucesso de uma equipe de rugby, já que esses dados são essenciais na determinação da posição dos atletas no jogo, maximizando, desta forma, o desempenho de todo o time (PRADO et al., 2006).

O presente estudo teve como objetivo avaliar o estado nutricional e consumo alimentar de atletas da equipe de Rugby de Guarapuava, PR.

\section{MATERIAIS E MÉTODOS}

Tipo de estudo

Estudo transversal com 21 jogadores da equipe masculina de Rugby Lobo Bravo de Guarapuava, PR, Brasil, com idade entre 19 e 37 anos.

\section{Questões éticas}

Os atletas assinaram um Termo de Consentimento Livre e Esclarecido (TCLE) e o projeto foi aprovado pelo Comitê de Ética em Pesquisa da UNICENTRO (Parecer 164/2010). Os critérios de exclusão foram indivíduos menores de idade e os que se recusaram a assinar o TCLE.

\section{Avaliação do estado nutricional}

Para a avaliação antropométrica foram aferidas as medidas de peso corporal $(\mathrm{kg})$ e estatura (m), de acordo com o preconizado pelo Ministério da Saúde (BRASIL, 2004). Foi utilziada uma balança eletrônica (Powerpack $^{\circledR}$, China) com capacidade 
máxima de $150 \mathrm{~kg}$ e escala de $100 \mathrm{~g}$, e uma fita métrica inelástica acoplada à parede sem rodapé. A partir desses valores, foi realizado o cálculo de índice de massa corporal (IMC) através da razão entre peso corporal atual $(\mathrm{kg})$ e o quadrado da estatura $\left(\mathrm{m}^{2}\right)$ dos atletas. A classificação e diagnóstico do estado nutricional (IMC) seguiu os pontos de corte para adultos (OMS, 1998).

O registro da circunferência do braço (CB) foi realizado por meio do cálculo de adequação e classificação segundo Blackburn e Thornton (1979). Com isso, foi calculada a circunferência muscular do braço (CMB) (FRISANCHO, 1981). A determinação de gordura corporal foi realizada através da medida das quatro principais dobras cutâneas (triciptal, biciptal, subescapular e suprailíaca) avaliadas com um adipômetro científico (Cescorf ${ }^{\circledR}$, Brasil), com precisão de $0,1 \mathrm{~mm}$, no lado não dominante dos indivíduos (LOHMAN, 1991). A classificação de porcentagem de gordura baseou-se na soma das quatro dobras subcutâneas (DURNIN; WOMERSLEY, 1974), e sua classificação foi de acordo com Lambert (2009) para jogadores de rugby onde o valor de referencia é $17 \%$ de gordura corporal.

Os atletas também foram submetidos à avaliação da gordura corporal por outros três métodos de bioimpedância: a) balança eletrônica digital (BBD) (Powerpack ${ }^{\circledR}$, China) com monitoramento de gordura corporal e escala de hidratação; b) bioimpedância bipolar (BB) com o aparelho Fitness Monitor $^{\circledR}$ (Modelo FE-068 Tech Line, Brasil) e; c) bioimpedância em disposição tetrapolar (BT) realizada com o aparelho Maltron ${ }^{\circledR}$ (BF907, Inglaterra), no lado direito do corpo onde foram colocados quatro eletrodos, dois nas mãos e dois nos pés com o indivíduo deitado, em decúbito dorsal (COSTA, 2002). Os participantes foram orientados a seguir todos os procedimentos pré-testes proposto por Lukaski et al. (1986).

\section{Avaliação dietética/socioeconômica}

Para a avaliação dietética foi aplicada uma anamnese (dados socioeconômicos, questionário de frequência alimentar e dia habitual alimentar), adaptada de Duarte (2007) e Ribeiro e Frank (1998). Foi utilizado o software Dietwin ${ }^{\circledR}$ (versão 2.0, 2008) para o cálculo de calorias e percentual de macro e micronutrientes.

Como valores de recomendação de calorias e macronutrientes, empregou-se a média do preconizado pela Sociedade Brasileira de Medicina do Exercício e do Esporte (SBMEE, 2009). Os valores de micronutrientes foram preconizados conforme Bernardot (2006), para atletas. A taxa metabólica basal (TMB) e o gasto energético total (GET) foram calculados conforme o preconizado pela IOM (2002). Para o cálculo do GET utilizou-se nível de atividade física 
1,25, considerada moderada por McArdle et al. (2011).

\section{Análise estatística}

Para análise dos dados utilizou-se o software Statistical Package for Social $5 \%(\mathrm{p}<0,05)$.

\section{RESULTADOS E DISCUSSÃO}

\section{Avaliação do estado nutricional}

$\mathrm{Na}$ Tabela 1 pode ser verificada a avaliação antropométrica, a \% de gordura corporal e de líquidos analisados pelos diferentes aparelhos de bioimpedância. Em estudo de Lopes et al. (2011), avaliando atletas de rugby, os backs apresentaram média de $76,3 \pm 6,9 \mathrm{~kg}$ e os Forwards de 96,3 $\pm 21,1$ $\mathrm{kg}$, sendo este último bem próximo ao encontrado na presente pesquisa. Com relação à altura dos atletas verificou-se média semelhante ao trabalho de Lopes et al. (2011) que encontraram média de $1,78 \mathrm{~m}$.

Quanto ao IMC foi encontrado o valor encontrado está dentro da faixa média relatada

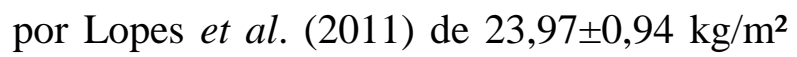
(Backs) e $30,16 \pm 5,18 \mathrm{~kg} / \mathrm{m}^{2}$ (Forwards). A média de $\mathrm{CB}$ mostrou-se próxima à preconizada de 31,97\% (BLACKBURN; THORNTON, 1979) dado que se assemelha ao estudo de Sousa et al. (1999), com atletas profissionais de futebol. Entretanto, a CMB
Sciences $\quad\left(\operatorname{SPSS}^{\circledR}\right)$ versão 19.0. Foram realizadas análises estatísticas descritivas, Qui-quadrado de Pearson, Correlação Simples de Pearson, teste $\mathrm{t}$ de Student e teste de Tukey, todos com nível de significância de apresentou-se superior a recomendação de 27,55\% (FRISANCHO, 1981).

Em relação à média da \% de gordura corporal, foi encontrado valor acima do recomendado (17\%), para todos os métodos avaliados. O método que mostrou maior valor de gordura corporal foi à bioimpedância tetrapolar. Resultado que corrobora com Gabbett (2000), avaliando atletas de rugby (17,5 a 19,9\%). Lopes et al. (2011) também encontraram valores de $30 \%$ de gordura corporal para Forwards, porém para os Backs foi notada valores adequados $(14,8 \%)$.

$\mathrm{Na}$ análise da \% de líquido corporal, o maior valor encontrado foi na bioimpedância em balança digital, estando abaixo da média recomendada (58\%), indicada pelo aparelho de avaliação de bioimpedância tetrapolar. Esse fato pode ser explicado devido à maior tendência dos atletas de rugby para perda de líquidos e eletrólitos durante uma partida, principalmente pelo suor. Destaca-se que o atleta não pode depender da sede para iniciar a ingestão hídrica, pois a hiperidratação protege contra o estresse térmico (PERRELA et al., 2005). 
Tabela 1 - Dados antropométricos, \% de gordura corporal e de líquidos dos atletas do time de rugby, Guarapuava, PR

\begin{tabular}{lrr}
\hline Variáveis & Média & Desvio padrão \\
\hline Peso $(\mathrm{kg})$ & 90,35 & 14,89 \\
Altura (m) & 1,79 & 0,06 \\
IMC $\left(\mathrm{kg} / \mathrm{m}^{2}\right)$ & 28,26 & 3,50 \\
Circunferência do Braço (cm) & 32,81 & 3,33 \\
Adequação da Circunferência do Braço (\%) & 104,88 & 9,46 \\
Circunferência Muscular do Braço (cm) & 30,79 & 3,24 \\
Adequação da Circunferência Muscular do Braço (\%) & $11,54,08$ \\
Prega Cutânea Triciptal (mm) & 9,54 & 2,21 \\
Prega Cutânea Biciptal (mm) & 6,43 & 2,27 \\
Prega Cutânea Subescapular (mm) & 16,14 & 4,62 \\
Prega Cutânea Suprailíaca (mm) & 17,00 & 5,79 \\
\% de gordura corporal pelas 4 pregas cutâneas & 18,64 & 3,27 \\
Bioimpedância bipolar (\% de gordura) & 19,16 & 6,07 \\
Bioimpedância tetrapolar (\% de gordura) & 34,06 & 7,60 \\
Bioimpedância - balança digital (\% de gordura) & 24,05 & 4,14 \\
Bioimpedância tetrapolar (\% de líquidos) & 47,73 & 5,75 \\
Bioimpedância com balança digital (\% de líquidos) & 52,16 & 2,85 \\
\hline
\end{tabular}

Fonte: os autores.

Na Tabela 2 é apresentada a classificação do estado nutricional dos atletas, conforme o IMC, CB, CMB e porcentagem de gordura corporal pelo método da soma das quatro dobras subcutâneas. Verificou-se que a maioria dos indivíduos avaliados encontra-se com sobrepeso, segundo o estado nutricional para o IMC. Resultados distintos foram relatados por Lopes et al. (2011), analisando jogadores de rugby. Os atletas Backs e Forwards classificaram-se, em geral, com eutrofia e obesidade grau 1, respectivamente. É importante salientar que a classificação de atletas pelo IMC não é a mais recomendada, já que esse método não diferencia a composição corporal em massa gorda e massa livre de gordura, daí a necessidade de diferentes avaliações antropométricas concomitantes (ANJOS, 1992).

A avaliaçõa da CMB mostrou $100 \%$ de eutróficos, número menor que a classificação da CB. Já, pela avaliação da \% de gordura corporal, observou-se que a maioria dos atletas apresentava porcentagem superior ao preconizado.

Tabela 2 - Classificação do estado nutricional conforme o IMC, circunferência do braço e porcentagem de gordura corporal dos atletas do time de rugby, Guarapuava, PR

\begin{tabular}{lc}
\hline Classificação do IMC* & Percentual (\%) (n) \\
\hline Eutrofia & $28,5(6)$ \\
Sobrepeso & $42,9(9)$ \\
Obesidade grau I & $28,6(6)$ \\
\hline Classificação da CB* & $76,2(16)$ \\
\hline Eutrofia & $14,3(3)$ \\
Sobrepeso & $9,5(2)$ \\
Obesidade & $100(21)$ \\
\hline Classificação da CMB* & $38,1(8)$ \\
\hline Eutrofia & $61,9(13)$ \\
\hline Classificação da \% GC* & \\
\hline Normal & \\
Elevado & IMC: índice de massa corporal; CB: circunferência do braço; GC: gordura corporal avaliada pelo método da soma das quatro dobras subcutâneas.
\end{tabular}


Com o objetivo de analisar o grau de associação entre os métodos utilizados na avaliação da \% de gordura e líquido corporal, calculou-se o coeficiente de correlação de
Pearson (r), juntamente com o erro padrão de estimativa (EPE). Os resultados podem ser visualizados na Tabela 3.

Tabela 3 - Correlação de Pearson (r) e erro padrão de estimativa (EPE) do percentual de gordura e líquidos para os aparelhos de bioimpedância referência, bioimpedância tetrapolar e bioimpedância bipolar

\begin{tabular}{|c|c|c|c|}
\hline & $\begin{array}{c}\text { Método de referência } \\
\% \text { gordura }\end{array}$ & $\mathrm{BBD}$ & $\mathrm{BB}$ \\
\hline \multicolumn{4}{|c|}{ BT } \\
\hline $\mathbf{R}$ & & $0,76^{*}$ & $0,82^{*}$ \\
\hline $\mathbf{p}$ & & $\mathrm{p}=0,00$ & $\mathrm{p}=0,00$ \\
\hline EPE & & 5,04 & 4,36 \\
\hline \multicolumn{4}{|c|}{ BB } \\
\hline $\mathbf{R}$ & & $0,79^{*}$ & - \\
\hline $\mathbf{p}$ & & $\mathrm{p}=0,00$ & \\
\hline EPE & & 3,80 & \\
\hline & \% líquidos & & - \\
\hline \multicolumn{4}{|c|}{ BT } \\
\hline $\mathbf{R}$ & & $0,60^{*}$ & - \\
\hline $\mathbf{p}$ & & $\mathrm{p}=0,00$ & \\
\hline EPE & & 2,32 & \\
\hline
\end{tabular}

"Correlação estatisticamente significativa $(\mathrm{p}<0,05)$ realizada pela Correlação de Pearson $(r)$; EPE: Erro Padrão de Estimativa; BBD: Bioimpedância com balança digital; BT: bioimpedância tetrapolar; BB: Bioimpedância Bipolar. Fonte: os autores.

Todos os métodos apresentaram fortes correlações positivas e significantes com o método de referência, tanto na \% de gordura como na \% de líquidos, sendo maior com a bioimpedância bipolar e menor com a bioimpedância com balança digital. Para os líquidos, também houve forte correlação $(\mathrm{p}<0,05)$.

Segundo a classificação de Lohman (1991), o EPE deve ser < 2,0\% para qualificar um método como ideal; até $2,5 \%$ para ser considerado como excelente; até $3,0 \%$ para ser muito bom; até $3,5 \%$ para ser avaliado como bom; até $4,0 \%$ para ser razoavelmente bom; até $4,5 \%$ fraco e, por fim, até $5,0 \%$ sendo classificado como não recomendado. Assim, apesar das correlações para \% de gordura apresentadas no presente trabalho serem fortes, nenhum método apresentou EPE $<3,5 \%$ classificado como "bom". Entretanto, a relação referência BB com BBD qualificouse como um método razoavelmente bom, já o método de referência BT com a BB apresentou classificação fraca.

Em estudo de Oliveira et al. (2010), avaliando diversas equações para estimativa de gordura corporal em indivíduos adultos, todos os métodos avaliados apresentaram fortes correlações significativas, o que se assemelha ao presente trabalho. Entretanto, dois dos cinco métodos analisados pelos autores apresentaram EPE $<3,5 \%$ (bom), diferente do presente estudo. O mesmo ocorreu em Rodrigues et al. (2001), avaliando 
a estimativa de gordura por diferentes métodos. Dois dos seis aparelhos analisados apresentaram-se com EPE $<3,5 \%$. Contudo, nem todos os métodos apresentaram fortes correlações significativas, sendo que dois deles não obtiveram significância.

Quanto à \% de líquidos, o método avaliado se mostrou excelente, EPE $<2,5 \%$, apresentando forte correlação significativa.

\section{Avaliação dietética/socioeconômica}

A média de idade encontrada na avaliação dos atletas foi de $24,67 \pm 4,8$ anos.

$\mathrm{Na}$ Tabela 4 estão descritas as informações

socioeconômicas

$\mathrm{e}$

complementares da amostra avaliada. O nível de escolaridade prevalente foi o ensino superior incompleto. Dos 21 atletas avaliados, 17 afirmaram não ter o hábito de fumar, 12 não consumiam bebidas alcoólicas regularmente e 90,5\% não faziam uso de suplementos. Resultados semelhantes aos relatados por Sartori et al. (2002), com atletas de futsal do estado do Rio Grande do Sul $(n=10)$ e do estado do Paraná $(n=11)$, que não faziam uso do cigarro, porém a maioria dos avaliados consumia bebidas alcoólicas regularmente.

Tabela 4 - Dados socioeconômicos e complementares de 21 atletas do time de rugby, Guarapuava, PR

\begin{tabular}{lc}
\hline Características & Frequência (\%) $(n)$ \\
\hline Curso técnico & $4,8(1)$ \\
Ensino médio completo & $14,3(3)$ \\
Superior completo & $19,0(4)$ \\
Superior incompleto & $47,6(10)$ \\
Pós-graduação & $14,3(3)$ \\
Uso de medicação & $9,5(2)$ \\
Uso de suplementos & $9,5(2)$ \\
Tabagista & $19,0(4)$ \\
Uso de bebidas alcoólicas & $42,9(9)$ \\
Prática de exercícios além do treino & $57,2(12)$ \\
\hline
\end{tabular}
Fonte: os autores.

Os valores médios da TMB e GET foram $1.921,77 \pm 171,23 \quad$ kcal e $3.418,10 \pm 321,63 \mathrm{kcal}$, respectivamente. Considerando que a média ideal é de 40 $\mathrm{kcal} / \mathrm{kg} / \mathrm{dia}$ (SBMEE, 2009) e a média de peso dos atletas avaliados foi de $90,35 \mathrm{~kg}$, verificou-se um valor médio de $3.614 \mathrm{kcal}$ de GET ideal, podendo-se concluir assim, que a dieta dos atletas encontrou-se abaixo do valor recomendado. Resultados semelhantes foram relatados por Prado et al. (2006) e Sartori et al. (2002), com jogadores de futebol e futsal, respectivamente.

A Figura 1 mostra a frequência do consumo de alimentos e grupos alimentares relevantes na dieta de atletas de rugby do município de Guarapuava, PR. Destaca-se a elevada frequência no consumo de café, carnes vermelhas, leguminosas, leite e derivados. As frutas foram informadas como 
ingeridas diariamente, por pouco mais de $50 \%$ dos indivíduos avaliados. Já as hortaliças cruas, são bastante consumidas pelos atletas (80\%). Em contrapartida a ingestão de suplementos orais não se mostra muito frequente, porém essa prática é muito comum em indivíduos que realizam atividade física (SBMEE, 2009).

Figura 1 - Frequência do consumo de alimentos e grupos alimentares consumidos pelos atletas do time de rugby, Guarapuava, PR.

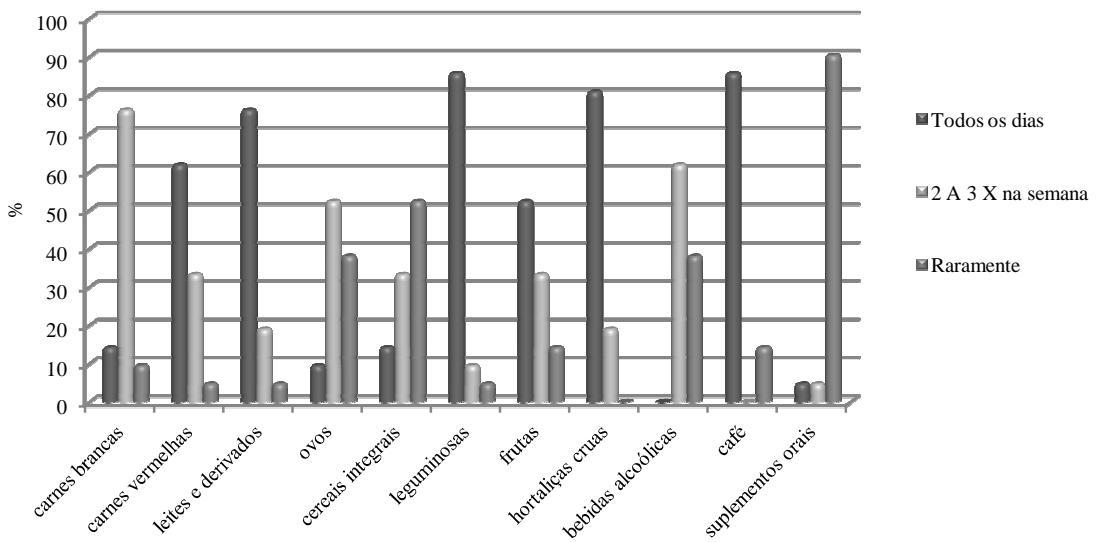

Fonte: os autores.

$\mathrm{Na}$ Tabela 5 verifica-se a média da ingestão de energia, macro e micronutrientes, conforme o relato do dia alimentar habitual, comparada com a recomendação desses nutrientes para atletas (BERNARDOT, 2006; SBMEE, 2009). Os valores de calorias e carboidratos apresentaram média de ingestão menores $(p<0,05)$ que os sugeridos, corroborando com Müller et al. (2007) e Quintão et al. (2009), analisando o hábito alimentar de jogadores de futebol.

Tabela 5 - Média de ingestão diária habitual e recomendação de energia, macro e micronutrientes dos atletas do time de rugby avaliados, Guarapuava, PR

\begin{tabular}{|c|c|c|c|}
\hline Nutriente & Média de ingestão $\pm \mathrm{DP}$ & Média preconizada** & $\mathbf{p}$ \\
\hline Calorias (kcal/kg/dia) & $38,20 \pm 2,75$ & 40,00 & $0,00^{*}$ \\
\hline Carboidratos (\%/dia) & $55,11 \pm 8,64$ & 60,00 & $0,01 *$ \\
\hline Proteínas (\%/dia) & $17,84 \pm 4,26$ & 15,00 & $0,00^{*}$ \\
\hline Lipídeos (\%/dia) & $27,03 \pm 7,51$ & 25,00 & 0,23 \\
\hline Cálcio (mg/dia) & $725,41 \pm 364,65$ & $1.300,00$ & $0,00 *$ \\
\hline Ferro (mg/dia) & $21,84 \pm 10,91$ & 15,00 & $0,00 *$ \\
\hline Zinco (mg/dia) & $13,65 \pm 6,47$ & 11,00 & 0,07 \\
\hline Vitamina A (mcg/dia) & $543,72 \pm 782,15$ & 900,00 & $0,04 *$ \\
\hline Vitamina C (mg/dia) & $81,29 \pm 84,48$ & 200,00 & $0,00 *$ \\
\hline Vitamina E (mg/dia) & $13,43 \pm 9,23$ & 15,00 & 0,43 \\
\hline
\end{tabular}

A ingestão de carboidratos é antes, durante e após o exercício físico, fundamental para a recuperação do atleta atuando na restauração dos estoques 
musculares e hepáticos de glicogênio, reposição de eletrólitos além de regeneração de lesões que podem ser causadas pelo exercício, devido a isso, seu consumo adequado, principalmente para atletas, tornase essencial, resultando em melhora do desempenho físico (GUERRA et al., 2001).

Os resultados de proteínas mostraramse maiores que os preconizados pela SBMEE (2009), corroborando com Quintão et al. (2009). Este fato pode ser justificado, uma vez que as necessidades protéicas devem ser atendidas integralmente por indivíduos praticantes de atividade física, já que participam, principalmente, no reparo de microlesões musculares, que ocorrem durante os jogos. Atletas de rugby, em especial, necessitam de um consumo adequado de proteínas, por se tratar de um esporte de alta intensidade. Porém, devem ser conscientizados de que a ingestão excessiva não eleva a massa magra, existindo um limite para seu acúmulo no organismo, o que pode sobrecarregar, principalmente, a função renal e hepática (VIEBIG; NACIF, 2006; SBMEE, 2009).

Já, com relação aos teores de lipídios, verifica-se que não houve diferença estatística entre a ingestão dos atletas e a recomendação. Durante exercícios intensos, a mobilização de ácidos graxos é mais acentuada. É importante ressaltar que, conforme citado por Oliveira e Marins (2008), o elevado consumo de lipídeos é muito comum em atletas, o que discorda dos dados observados no trabalho em questão. Os autores enfatizam ainda, que esse nutriente é essencial na produção de energia, além de transportar vitaminas lipossolúveis e compor a membrana celular.

As Vitaminas A e C obtiveram resultados de ingestão inferiores $(p<0,05)$ ao recomendado (BERNARDOT, 2006), entretanto a os atletas apresentaram ingestão adequada de vitamina E. Sartori et al. (2002) avaliando o consumo alimentar de atletas de futsal, também verificaram baixo consumo de Vitamina A.

O cálcio apresentou média de ingestão inferior à preconizada $(\mathrm{p}<0,05)$, concordando com estudos de Sartori et al. (2002). Entretanto, os valores de ferro foram superiores ao recomendado. Não houve diferença significativa no consumo de zinco.

Conforme explicam Guerra et al. (2001), os atletas estão sempre em elevado risco de ingestão inadequada de nutrientes, tanto de vitaminas quanto de minerais, já que se exercitam sob alta intensidade e por longos períodos de tempo. Isso pode ser observado com os atletas de rugby estudados, que apresentaram inadequação na ingestão da maioria dos micronutrientes avaliados (cálcio, ferro, vitamina A e vitamina $C$ ), somando-se a isso, observou-se, também, o baixo consumo de frutas na avaliação da frequência alimentar (Figura 1). Alguns desses nutrientes 
apresentam funções importantes no metabolismo, composição muscular e óssea, além de prevenir a oxidação lipídica. Assim, quando há uma inadequação no consumo nutricional, o comprometimento da capacidade aeróbica e anaeróbica é muito comum, enfatizando-se a necessidade da ingestão adequada desses alimentos/nutrientes.

\section{CONCLUSÃO}

Quanto ao estado nutricional, verificou-se que a maioria dos atletas encontra-se com percentual de gordura corporal superior à recomendação.

O método de bioimpedância tetrapolar foi melhor correlacionado com a bioimpedância bipolar, sendo os mais adequados para avaliação da porcentagem de gordura dos atletas.

Em geral, observaram-se valores inadequados na ingestão de macro e micronutrientes.

\section{REFERÊNCIAS}

ANJOS, L.A. Índice de massa corporal (massa corporal.estatura-2) como indicador do estado nutricional de adultos: Uma revisão da literatura. Revista de Saúde Pública, v.26, n.6, p.431-436, 1992.

\section{BERNARDOT, D. Advanced Sport}

nutrition. 2006. Disponível em: <http://www. healthline .com/hlbook/ advanced-sportsnutrition>. Acesso em: 01/01/2015.

BLACKBURN, G.L.; THORNTON, P.A. Nutritional assessment of the hospitalized patients. The Medical clinics of North America, v.63, n.5, p.11103-11115, 1979. BRASIL. Ministério da Saúde. Vigilância Alimentar e Nutricional - SISVAN.

Orientações básicas para a coleta, o processamento, a análise de dados e a informação em serviços de saúde. Série A. Normas e Manuais Técnicos. $1^{\text {a }}$ ed. Brasília (DF): Ministério da Saúde, 2004.

CABRAL, C.A.C.; ROSADO G.P.; SILVA, C.H.O.; MARINS, J.C.B. Diagnóstico do estado nutricional dos atletas da Equipe Olímpica Permanente de Levantamento de Peso do Comitê Olímpico Brasileiro (COB). Revista Brasileira de Medicina do Esporte, v.12, n.6, p.345-350, 2006.

CONFEDERAÇÃO BRASILEIRA DE RUGBY. Disponível em: <http://www. brownbag.com.br/brasilrugby/v1/index. asp>. Acesso em: 03/01/2015.

COSTA, R.P. Avaliando a composição corporal. In: HIRSCHBRUCH, M.D.; CARVALHO, J.R. (Org.). Nutrição esportiva: uma visão prática. São Paulo (SP): Manole, 2002. 
DUARTE, A.C.G. Avaliação nutricional: aspectos clínicos e laboratoriais. São Paulo (SP): Atheneu, 2007.

DURNIN, J.V.; WOMERSLEY, J. Body fat assessed from total body density and its estimation from skinfold thickness: measurements on 481 men and women aged from 16 to 72 years. British Journal of Nutrition, v.32, n.1, p.77-97, 1974.

FRISANCHO, A.R. New norms of upper limb fat muscle areas for assessment of nutritional status. American Journal of Clinical Nutrition, v.34, n.11, p.2540-2545, 1981.

\section{GABBETT, T.J. Physiological and} anthropometric characteristics of amateur rugby league players. British Journal of Sports Medicine, v.34, n.4, p.303-307, 2000.

GUERRA, I.; SOARES, E.A.; BURINI, R.C. Aspectos nutricionais do futebol de competição. Revista Brasileira de Medicina do Esporte, v.7, n.6, 200-206, 2001.

INSTITUTE OF MEDICINE (IOM). FOOD AND NUTRITION BOARD. Dietary reference intakes. Energy, carbohydrate, fiber, fat, fatty acids, cholesterol, protein and amino acids. Washington (DC): National Academy Press, 2002.

\section{LAMBERT, M.I. Aspects of physical} conditioning for rugby. South Africa (CT): University of Cape Town, 2009.

LOHMAN, T.G. Skinfold thicknesses and measurement technique. In: LOHMAN, T.G.; ROCHE, A.F.; MARTORELL, R. (Org.). Anthropometric standardization reference manual. Champaing (IL): Human Kinetics Books, 1991.

LOPES, A.L.; PINHEIRO, E.S.; CUNHA, G.S.; SAPATA, K.; MARTINS, J.B.; CARTERI, R.B.; RIBEIRO, G.S.; CARDOSO, M.S. Análise da composição corporal e da capacidade aeróbica em jogadores de rugby. EFDeportes.com, v.16, n.158, p.1, 2011.

LUKASKI, H.C.; BOLONCHUK, W.W.; HALL, C.B.; SIDERS, W.A. Validation of tetrapolar bioelectrical impedance method to assess human body composition. Journal of Applied Physiology, v.60, n.4, p.1327-1332, 1986.

MCARDLE, W.D.; KATCH, F.I.; KATCH, V.L. Composição corporal, equilíbrio energético e controle do peso. In: MCARDLE, W.D.; KATCH, F.I.; KATCH, V.L. (Org.). Fisiologia do Exercício: energia, nutrição e desempenho humano. 7 ed. Rio de Janeiro (RJ): Guanabara Koogan, 2011. 
MÜLLER, C.; ALVES, C.P.; ROSTIROLLA, L.; NAVARRO, A.C.; NAVARRO, F.

Avaliação do nutricional de jogadores de futebol. Revista Brasileira de Nutrição

Esportiva, v.1, n.1, p.30-39, 2007.

OLIVEIRA, G.T.C.; MARINS, J.C.B.

Práticas dietéticas em atletas: especial atenção ao consumo de lipídios. Revista Brasileira de Ciência e Movimento, v.16, n.1, p.77-88, 2008.

OLIVEIRA, J.S.; ROSADO, L.E.F.P.L.R.; ROSADO， G.P.; RIBEIRO， R.C.L.; FRANCESCHINI, S. C. C.; OLIVEIRA, J.C. Comparação de métodos para estimativa de gordura corporal de indivíduos adultos. EFDeportes.com, v.15, n.149, p.1, 2010.

ORGANIZAÇÃO MUNDIAL DA SAÚDE (OMS). Reunião Mista. Necessidades de energia e proteína. (OMS - Série de Relatos Técnicos, 724). São Paulo (SP): Roca, 1998.

PANZA, V.P.; COELHO, M.S.P.H.; DI

PIETRO, P.F.; ASSIS, M.A.A.;

VASCONCELOS, F.A.G. Consumo

alimentar de atletas: reflexões sobre recomendações nutricionais, hábitos alimentares e métodos para avaliação do gasto e consumo energéticos. Revista de Nutrição, v.20, n.6, p.681-692, 2007.

PERRELA, M. M.; NORIYUKI, P.S.; ROSSI, L. Avaliação da perda hídrica durante um treino intenso de rugby. Revista
Brasileira de Medicina do Esporte, v.11, n.4, p.229-232, 2005.

PRADO, W.L.; BOTERO, J.P.; GUERRA, R.L.F.; RODRIGUES, C.L.; CUVELLO, L.C.; DÂMASO, A.R. Perfil antropométrico e ingestão de macronutrientes em atletas profissionais brasileiros de futebol, de acordo com suas posições. Revista Brasileira de Medicina do Esporte, v.12, n.2, p.61-65, 2006.

QUINTÃO, D.F.; OLIVEIRA, G.C.; SILVA, S.A.; MARINS, J.C.B. Estado nutricional e perfil alimentar de atletas de futsal de diferentes cidades do interior de Minas Gerais. Revista Brasileira de Futebol, v.2, n.1, p.13-20, 2009.

RIBEIRO, B.G.; FRANK, A.A. Nutrição aplicada à Dietética - Bases para um planejamento alimentar. Rio de Janeiro (RJ): CC\&P Editores, 1998.

RODRIGUES, M.N.; SILVA, S.C.; MONTEIRO, W.D.; FARINATTI, P.T.V. Estimativa de gordura corporal através de equipamentos de bioimpedância, dobras cutâneas e pesagem hidrostática. Revista Brasileira de Medicina do Esporte, v.7, n.4, p.125-131, 2001.

SARTORI, R.F.; PRATES, M.E.F.; TRAMONTE, V.L.G.C. Hábitos alimentares de atletas de futsal dos estados do Paraná e do 
Rio Grande do Sul. Revista de Educação

Física, v.13, n.2, p.55-62, 2002.

SOCIEDADE BRASILEIRA DE

MEDICINA DO EXERCÍCIO E DO

ESPORTE (SBMEE). Diretrizes dietéticas:

Modificações dietéticas, reposição hídrica,

suplementos alimentares e drogas:

comprovação de ação ergogênica e potenciais

riscos para a saúde. Revista Brasileira de

Medicina do Esporte, v.15, n.3, p.1-12,

2009.

SOFTWARE DE AVALIAÇÃO

NUTRICIONAL. DIETWIN profissional

[programa de computador]. Versão 2.0.

Porto Alegre: Brubins Comércio de

Alimentos e Supergelados, 2008.

SOUSA, M.S.C.; SOUSA, S.J.G.; SANTOS,

J.P. O percentual de gordura em atletas

profissionais de futebol segundo diferentes

métodos: ensaio envolvendo condições

desportivas e de saúde. Revista Brasileira de

Atividade Física \& Saúde, v.4, n.3, p.64-74,

1999.

VIEBIG, R.F.; NACIF, M.A.L.

Recomendações nutricionais para a atividade

física e o esporte. Revista Brasileira de

Educação Física Esporte, Lazer e Dança,

v.1, n.1, p.2-14, 2006. 